\title{
A systematic approach to chronic heart failure care: a consensus statement
}

\section{Karen Page \\ RN, DN, BEd \\ National Manager, Health Equity and Secondary \\ Prevention \\ Thomas H Marwick \\ MBBS, PhD, MPH \\ Director $^{2}$ \\ Rebecca Lee \\ BPharm, MPH \\ Project Officer, Health Equity and Secondary Prevention \\ Robert Grenfell MBBS, MPH, FAFPHM National Director, \\ Cardiovascular Health}

Walter P Abhayaratna

FRACP, FACC, DrPH Cardiologist and Director of Clinical Trials $^{3}$

Anu Aggarwal MBBS, FRACP, PhD Cardiologist ${ }^{4}$

Tom G Briffa PhD

Jan Cameron PhD, MHSc(Health Prom\&HealthEd), BN Senior Research Fellow ${ }^{6}$

Patricia M Davidson RN, PhD Director ${ }^{7}$

Andrea Driscoll $\mathrm{PhD}$

Associate Professor ${ }^{8}$

Jacquie Garton-Smith MBBS, FRACGP Clinical Lead $^{9}$

Debra J Gascard BN, MNP, NP Chronic Disease - Heart Failure Nurse Practitioner ${ }^{10}$

Annabel Hickey MMSc(ClinEpi), BApp(OT) Statewide Heart Failure Services Coordinator ${ }^{11}$

Dariusz Korczyk FRACP, FCSANZ Cardiologist $^{12}$

Julie-Anne Mitchell MPH, BA, RN

Cardiovascular Health Director $^{13}$

Rhonda Sanders RN, MNSt Clinical Nurse Consultant and Representative of Australian

College of Critical Care Nursing ${ }^{14}$
C hronic heart failure (CHF) remains a major public health problem. CHF is not a static syndrome; individuals with $\mathrm{CHF}$ are at high risk of progressive cardiac dysfunction resulting in either sudden cardiac death or acute hospitalisation. Despite significant advances in CHF management, clinical outcomes are poor and associated with escalating health care costs. ${ }^{1}$ Worldwide, there are an estimated 23 million people living with CHF and 5.7 million new cases each year. ${ }^{2}$ With limited Australian data available, the Australian Institute of Health and Welfare has used overseas rates to suggest that 30000 patients are diagnosed with incident heart failure annually and 300000 people are living with CHF in Australia. ${ }^{3}$ The prevalence of CHF continues to rise as the population ages and survival from cardiovascular disease continues to improve.

Between 2006 and 2011, deaths from CHF in Australia rose by $20 \%{ }^{4}$ CHF prognosis remains poorer than that for common forms of cancer (in terms of individual survival and population life-years lost) for men and women. 5,6 Hospital separations for CHF increased by $24 \%$ between 2002-03 and 2011-12. ${ }^{7}$ In the 2007-08 financial year, CHF was a primary diagnosis in 45212 hospitalisations and a contributory diagnosis in 94599 hospitalisations. ${ }^{8}$

The annual cost of CHF in Australia has been estimated at over $\$ 1$ billion per year, with hospital care being the largest expenditure. ${ }^{9}$ A significant proportion of this cost is associated with preventable $\mathrm{CHF}$ readmissions. Readmissions within 30 days of discharge can be as high as $20 \%-27 \% .10,11$ Overall, reported rates for readmission with CHF within 3-12 months of initial discharge are between $29 \%$ and $49 \% .{ }^{12,13}$

Given the high rate of readmissions, there is profound potential to improve CHF-related outcomes, at both individual and societal levels, through improved quality of care and system change. ${ }^{14}$

\section{Practice gaps}

Recent studies have highlighted significant variations in access to evidence-based care for patients with CHF. ${ }^{15-17}$ Many individuals are not diagnosed in a timely manner and subsequent management is suboptimal. Initial diagnostic delay is often due to under-recognition of early heart failure symptoms. This is compounded by limited availability of public sector services, particularly in rural and remote areas. Additionally, patient data are not shared across health services, largely due to lack of integrated information systems and care coordination.

\section{Summary}

The National Heart Foundation of Australia assembled an expert panel to provide guidance on policy and system changes to improve the quality of care for people with chronic heart failure (CHF). The recommendations have the potential to reduce emergency presentations, hospitalisations and premature death among patients with $\mathrm{CHF}$.

Best-practice management of $\mathrm{CHF}$ involves evidencebased, multidisciplinary, patient-centred care, which leads to better health outcomes. A CHF care model is required to achieve this.

Although CHF management programs exist, ensuring access for everyone remains a challenge. This is particularly so for Aboriginal and Torres Strait Islander peoples, those from non-metropolitan areas and lower socioeconomic backgrounds, and culturally and linguistically diverse populations.

Lack of data and inadequate identification of people with CHF prevents efficient patient monitoring, limiting information to improve or optimise care. This leads to ineffectiveness in measuring outcomes and evaluating the $\mathrm{CHF}$ care provided. Expanding current cardiac registries to include patients with $\mathrm{CHF}$ and developing mechanisms to promote data linkage across care transitions are essential.

As the prevalence of $\mathrm{CHF}$ rises, the demand for multidisciplinary workforce support will increase. Workforce planning should provide access to services outside of large cities, one of the main challenges it is currently facing.

To enhance community-based management of CHF, general practitioners should be empowered to lead care. Incentive arrangements should favour provision of care for Aboriginal and Torres Strait Islander peoples, those from lower socioeconomic backgrounds and rural areas, and culturally and linguistically diverse populations.

Ongoing research is vital to improving systems of care for people with CHF. Future research activity needs to ensure the translation of valuable knowledge and highquality evidence into practice.

These problems are amplified among marginalised populations. $\mathrm{CHF}$ is 1.7 times more common, and occurs at a younger age, among Aboriginal and Torres Strait Islander peoples than among other Australians. ${ }^{18}$ Aboriginal and Torres Strait Islander peoples are also more likely to die from $\mathrm{CHF}$, and their rate of preventable $\mathrm{CHF}$-related hospitalisations is three times higher than for non-Indigenous Australians. ${ }^{18}$ Such health disparities frequently occur due to poor access to evidence-based care. Availability 


\section{Consensus development process}

The National Heart Foundation of Australia convened an expert panel to provide guidance on policy and system changes to improve the quality of care for people with chronic heart failure. A relevant literature search was performed, limited to evidence from human studies published in English between 2003 and 2013. This was complemented with hand searching of reference lists from reviews and personal collections of the expert panel, and additional peer-review. As there is limited evidence around the system changes required, these consensus recommendations are based on expert opinion. They are not exhaustive, and many other changes and actions can be implemented by both individuals and organisations to improve care outcomes. The recommendations are generally broad, rather than prescriptive, and many can be implemented with minimal resourcing.

of culturally appropriate services that provide earlier prevention, detection and management of $\mathrm{CHF}$ needs to be improved.

Among people hospitalised with $\mathrm{CHF}$, those who receive evidence-based, multidisciplinary care have better health outcomes than those who do not. ${ }^{19,20}$ Current Australian guidelines articulate the evidence-based practices necessary to improve care delivery. ${ }^{21}$ However, the management of CHF remains a pressing concern, with many apparent indicators of poor case detection, non-guideline-based management, poor coordination and communication, and recurrent hospital admission. ${ }^{15-17,22}$

In this consensus statement, our aim is to guide the policy and associated system changes required to support delivery of evidence-based care. This is not intended as a prescriptive guideline, rather a set of principles to assist health departments, health network administrators, clinicians and consumers in improving care systems for people living with CHF. Our intended audience is policymakers, health system managers, consumers and health professionals in acute and primary care, including cardiologists, general practitioners, nurses, dietitians and other members of the multidisciplinary team. The consensus development process is outlined in Box 1.

The expert panel identified four themes and five principles to inform the consensus statement (Box 2). The recommendations (Box 3 ) based on these themes have the potential to reduce the likelihood of emergency presentations, hospitalisations and premature death among patients with CHF.

\section{Chronic heart failure care model}

Best-practice management of CHF involves evidence-based, multidisciplinary, patient-centred care, which leads to better health outcomes. ${ }^{20,23}$ Patient-centred care is respectful of and responsive to preferences, needs and values of patients and consumers and should include dimensions of respect, emotional support, physical comfort, information and communication, continuity and transition, care coordination, involvement of family and carers, and access to care. ${ }^{23}$ Greater care coordination is needed because fragmentation across health care, long-term care and other social support systems effectively impedes a patient-centred focus. ${ }^{24}$ Research suggests that providing incentive payments through primary care payment schemes may improve care continuity and transition, as would streamlining funding for delivery of different levels of care. ${ }^{24}$
Deborah Spicer MNg(NP), GradDip(CVNursing), Heart Failure Clinical Practice Consultant and Heart Failure Nurse Practitioner ${ }^{15}$

Simon Stewart

Head of Preventative Health and Director. NHMRC Centre for Research Excellence to Reduce Inequality in Heart Disease ${ }^{16}$

Vicki Wade

DipAppSci, BHS, MN Cultural Lead, National Aboriginal Health Unit ${ }^{13}$

1 National Heart Foundation of Australia Melbourne, VIC

2 Menzies Research Institute Tasmania, Hobart, TAS.

3 Canberra Hospital Canberra, ACT.

4 Royal Melbourne Hospital, Melbourne, VIC

5 School of Population Health, University of Western Australia Perth, WA.

6 Cardiovascular Research Centre Australian Catholic University, Melbourne, VIC

7 Centre for Cardiovascular and Chronic Care, University of Technology Sydney, Sydney, NSW.

8 Faculty of Health Deakin University, Melbourne, VIC.

9 Cardiovascular Health Network, Department of Health Western Australia, Perth, WA.

10 Heart Failure Care, Monash Health, Melbourne, VIC.

11 Advanced Heart Failure and Cardiac Transplant Unit, The Prince Charles Hospital, Brisbane, QLD.

12 Heart Failure Unit, Princess Alexandra Hospital, Brisbane, QLD.

13 National Heart Foundation of Australia, Sydney, NSW.

14 Department of Cardiology, St Vincent's Hospital Melbourne Melbourne, VIC.

15 Community Heart Failure Nursing Southern Adelaide Local Health Network Adelaide, SA.

16 Baker IDI Heart and Diabetes Institute, Melbourne, VIC.

karen.page@ heartfoundation.org.au

doi: 10.5694/mjal4.00032
Multidisciplinary CHF care is distinguishable from generic chronic disease management programs by the special needs of these patients, which necessitate specialised evidence-based treatment strategies associated with optimal outcomes. ${ }^{25,26}$ Considerations include management of severely ill CHF patients, symptom monitoring, implementation of a range of self-management strategies and titration of medications.

Against a background of recent national health reform linking improved person-centred care with performance and funding arrangements, there is increasing interest in how to realign care systems accordingly. Research suggests that individuals value easy access to services, coordinated care, and information and honesty about their prognosis. ${ }^{27,28}$ Patient or consumer charters and informed consent policies have been introduced, but there is limited evidence that consumer engagement influences change in care delivery. ${ }^{29}$ Consumer engagement has been strengthened by the recent introduction of the National Safety and Quality Health Service Standards, which include a component on consumer partnership. ${ }^{30}$ The Standards deliver a framework that health organisations can use to actively engage and partner with consumers to strengthen health service delivery. ${ }^{30}$

Research suggests care coordination problems are greatest at the interfaces between health care sectors and between providers. ${ }^{24}$ Multidisciplinary care can overcome some of these barriers, as can pooling resources between sectors for care coordinators. ${ }^{24}$ Multidisciplinary program delivery needs to be appropriate to local needs, resources, patient preferences and disease trajectory phase, as well as across a range of delivery models, including home-based, clinic-based and telephone-based approaches, or a hybrid of these. $17,31,32$

CHF management plans that include a multidisciplinary approach are vital to educate and empower individuals and their carers to manage this challenging condition. ${ }^{11,33}$ Given limited resources, a risk assessment tool that stratifies patients at higher risk of readmission could be used to ensure those most likely to benefit from a management program are targeted. ${ }^{34}$ These plans should be clear about responsibilities among health care providers.

There are cardiac clinical networks in most Australian states and territories (Queensland, New South Wales, Victoria, South Australia, Western Australia, Northern Territory) that have championed access to evidence-based care for CHF patients. These networks have significant influence in improving care systems and outcomes, and can evaluate variance in care quality within and across jurisdictions, with the authority to develop funding models, including care packages. They can facilitate improvements in CHF care by fostering awareness, communication, partnerships and links; by engaging leaders across sectors; and by providing advice and advocacy for policy, planning and funding. The expert panel identified the clinical networks' essential role in ensuring systematic delivery of a multidisciplinary care model, and concluded this role should be strengthened and further developed. Although multidisciplinary CHF management programs exist across Australia, ensuring access for all patients who would benefit remains a challenge. This is particularly so 


\section{Themes and principles to reduce emergency presentations, hospitalisations and premature death among patients with chronic heart failure (CHF) \\ Theme Principle \\ CHF care model \\ Current evidence clearly identifies that accessible, multidisciplinary, guideline-based CHF care improves outcomes. \\ Access to meaningful data for management and benchmarking Collecting outcome data is the only accurate way of determining the effectiveness and cost of individual treatments; practice standards can then be based on up-to-date comparative effectiveness research. \\ Adequate patient information is a prerequisite for reducing unnecessary hospital admissions and medical errors. \\ Workforce An appropriately trained workforce with access planning to specialist cardiology support can deliver evidence-based care. \\ Research Research is essential to ensure an evidence base.}

for Aboriginal and Torres Strait Islander peoples, those from non-metropolitan areas and lower socioeconomic backgrounds, and culturally and linguistically diverse populations. ${ }^{16,35}$

Implementation of patient-centred care approaches can also have clinical and operational benefits, through less frequent readmissions and improved clinician and patient satisfaction. ${ }^{36-38}$ Other benefits include reduced emergency department re-presentations, fewer medication errors, higher functional status and improved evidence-based clinical care. ${ }^{38,39}$ There continues to be a large degree of heterogeneity between $\mathrm{CHF}$ programs, ${ }^{40}$ with some delivering high-quality complex care and others a simplistic program with minimal interventions. Research has shown that this has an impact on patient outcomes, ${ }^{41}$ and national guidelines have been developed to reduce this heterogeneity in Australia. ${ }^{21}$ Minimum accreditation standards are important for assessing multidisciplinary care services and reporting on best practice. ${ }^{16,42}$

\section{Access to meaningful data for management and benchmarking}

There is a paucity of Australian data on $\mathrm{CHF}$, resulting in reliance on extrapolation of overseas research. Lack of identification of people with symptomatic CHF prevents efficient patient monitoring. Expansion of cardiac registries to include patients with CHF could improve identification. Recall between health care providers to ensure appropriate assessments and treatments are completed at pre-agreed intervals is also often uncoordinated. An electronic health record potentially offers the ideal tool to track, document and supply $\mathrm{CHF}$ patients or their carers and health care providers with the appropriate health care information, on demand, to optimise care.

Further, we do not have standardised outcomes to measure and evaluate care effectiveness and enable international and national benchmarking activity. The definition of a quality indicator must be specific, complete, clearly worded and verified across different user groups. ${ }^{43}$ Another barrier to measuring standardised outcomes is poor data system compatibility across and within health services, which prevents efficient transfer of data and results in duplication of patient data collection. These problems could be reduced through the use of better process measures.

Increasingly, hospital readmission is becoming an important indicator of health care outcomes, as it can be used to identify potentially preventable admissions. However, as a sole indicator, it can be problematic due to difficulties with interpretation, utility in the clinical environment, and problems such as poor attendance at outpatient clinics, which does not necessarily reflect poor hospital care. An operational definition for readmission needs to clearly identify the diagnosis-related group or major disease classification associated with the index admission.

Over a longer period, as readmissions occur due to the chronic nature of the disease, event-free survival provides a measure of quality. ${ }^{44}$ An event is defined as an emergency presentation, hospitalisation or premature death within any 12 -month period. ${ }^{45}$

\section{Workforce planning}

Workforce needs are likely to be driven by the ageing population and associated disability rates, as well as changing technology, increased burden of disease and community expectations. ${ }^{46}$

One of the main challenges to workforce planning is providing access to services outside large cities. In 2006, Australian capital cities hosted $93 \%$ of CHF management programs, despite $40 \%$ of the known population with $\mathrm{CHF}$ living outside these cities. ${ }^{47}$ Policies that guide specialisation or multiskilling in the health workforce will become increasingly important. ${ }^{48}$

GPs should be empowered to lead care for patients with $\mathrm{CHF}$. This may be through the introduction of funding incentives or provision of nurse practitioners and practice nurses in primary care. Any incentive arrangements should favour provision of care for Aboriginal and Torres Strait Islander peoples, those from lower socioeconomic backgrounds and rural areas, and culturally and linguistically diverse populations. ${ }^{49}$

\section{Research}

Future research activity needs to build in processes to ensure the dissemination and translation into practice of valuable knowledge; the creation of ethical and evidencebased research policies; and the promotion, monitoring and implementation of high-quality health research evidence.

Research and quality improvement activity priorities arising from this consensus statement are those relating to CHF care models (including development of readmission risk assessment models), access to meaningful data for management and benchmarking, and workforce planning. Focused investigative teams, such as clinical $\mathrm{CHF}$ research networks, could lead this work. In addition, more work needs to be undertaken among populations for whom frequent access to mainstream services is limited; namely Aboriginal and Torres Strait Islander peoples, those from non-metropolitan areas and lower socioeconomic backgrounds, and culturally and linguistically diverse populations. 
Individuals with CHF have specific management needs. Future research should consider roles of specialty care teams (eg, cardiology, general medical) and the role of telehealth.

\section{Conclusion}

The current and future burden of CHF compels us to strive for equitable outcomes for all Australians. A national policy framework, with agreement between states, territories and the federal government, needs to be developed and implemented to tackle the increasing burden of CHF. Governments at national and state levels, together with cardiac clinical networks, need to ensure that evidencebased care models for people with $\mathrm{CHF}$ are standardised, with equitable access.

The core principles and recommendations described in this consensus statement should be incorporated into the various $\mathrm{CHF}$ systems of care operating across states and territories. Implementing these recommendations has the potential to improve the quality of care provided to individuals with $\mathrm{CHF}$, reducing associated costs for both the individual and the community. Improvements could be seen not only in the care experienced by patients and their families, but also in clinical and operational benefits. Implementing multidisciplinary, patient-centred care approaches can shorten lengths of stay in hospital, reducing health care costs and improving clinician and patient satisfaction. ${ }^{22,44,45,50}$ In the longer term, other benefits of patient-centred care include reduced emergency department re-presentations, fewer medication errors, higher functional status and improved clinical care. ${ }^{40-42}$

These recommendations can empower health care providers and organisations, peak and government organisations, care regulators, education providers and consumers to improve health outcomes for patients with $\mathrm{CHF}$ and to reduce harm. This work needs to be underpinned by nationally recognised standards for outcome measurement that are universally recognised and easily applied in practice. Data systems need to support evidence-based decision making, while providing feedback relating to standardised performance measures. Our health care workforce needs to be equipped to deal with the increasing burden of disease associated with $\mathrm{CHF}$, with training, education and research around the delivery of multidisciplinary care in an increasingly complex environment.

These recommendations, if adopted, have the potential to facilitate and promote optimal and equitable health outcomes for all Australians diagnosed with CHF.

Acknowledgements: We thank Rohan Greenland for his contribution to the content of this document.

Competing interests: Thomas Marwick is supported in part by a National Health and Medical Research Council Partnership grant (1059738). Simon Stewart declares an unrestricted education grant from Novartis Pharmaceuticals Australia, provided to the Baker IDI Heart and Diabetes Institute, to undertake a burden of disease in Australia report. He also received consulting fees from Novartis and payment from Menarini Australia for his involvement on an advisory board relevant to the treatment of heart failure. The National Heart Foundation of Australia supported all authors to attend the expert panel meeting held in Melbourne, through payment of travel and accommodation costs.

Provenance: Not commissioned; externally peer reviewed.

1 Biermann J, Neumann T, Angermann CE, et al. Economic burden of patients with various etiologies of chronic systolic heart failure analyzed by resource use and costs. Int J Cardiol 2012; 156: 323-325.
3 Recommendations to achieve a systematic approach to chronic heart failure (CHF) care*

\section{CHF care model}

1. Through state and territory cardiac clinical networks, support health departments to continue leading the development of integrated local care systems and future national quality improvement strategies within and across health services. ${ }^{\dagger}$

2. Identify and implement mechanisms to champion the uptake of clinical practice guidelines and delivery of integrated CHF services, according to local population need, within and across health services. ${ }^{\dagger}$

3. Develop minimum standards for CHF multidisciplinary care, which can be used to accredit health services and recognise best-practice health services or networks.

4. Develop robust funding models and examine the role of funded care packages in CHF care.

5. Establish system protocols and pathways to ensure effective clinical handover and service coordination across care transitions, and to activate appropriate services according to clinical need for an exacerbation, emergency presentation, hospitalisation or palliation.

6. Streamline care processes to facilitate early diagnosis, selfmanagement and multidisciplinary care planning, including primary care involvement and appropriate access to palliative services.

7. Embed mechanisms to promote the rights of individuals and their carers to facilitate their active engagement with health professionals and care systems.

\section{Access to meaningful data for management and benchmarking}

8. Develop national data definitions for CHF.

9. Expand current cardiac registries to include patients with $\mathrm{CHF}$.

10. Develop mechanisms to promote data linkage across care transitions.

11. Trial an electronic health record for people diagnosed with $\mathrm{CHF}$, so all current and future health care providers could, with the individual's consent, have access to the same information where and when they need it.

12. Use 12-month event-free survival as an outcome measure nationally to evaluate effectiveness of care systems. Events would include emergency presentations, hospitalisations and premature death.

13. Establish a national mechanism for monitoring and reporting $\mathrm{CHF}$ care outcomes against a nationally recognised set of goals and standards.

14. Develop a national set of indicators and standards to evaluate, inform and improve systems of care.

\section{Workforce planning}

15. Develop the workforce capacity across hospital and community services to deliver evidence-based care, appropriate to the local population, as identified in Guidelines for the prevention, detection and management of chronic heart failure in Australia ${ }^{31}$ and

Multidisciplinary care for people with chronic heart failure: principles and recommendations for best practice..$^{21}$

16. Develop robust funding models for the delivery of these services.

17. Examine mechanisms to empower general practitioners and other health care professionals in primary care to deliver evidence-based care for people with CHF.

\section{Research}

18. Create investigative teams, such as clinical CHF research networks, with active consumer collaboration.

19. Investigate approaches to optimise care delivery for Aboriginal and Torres Strait Islander peoples, those from non-metropolitan areas and lower socioeconomic backgrounds, and culturally and linguistically diverse populations.

* Level of evidence: expert panel consensus judgement. † Health services include area health services, local hospital networks, primary care, Aboriginal community controlled health organisations, aged care services and other appropriate agencies. 
2 Bui AL, Horwich TB, Fonarow GC. Epidemiology and risk profile of heart failure. Nat Rev Cardiol 2011; 8: 30-41.

3 Field B. Heart failure... what of the future? Australian Institute of Health and Welfare Bulletin No. 6. Cat. No. AUS 34. Canberra: AlHW, 2003. http:// www.aihw.gov.au/publication-detail/?id=6442467471 (accessed May 2013).

4 Australian Bureau of Statistics. Causes of death, Australia, 2011. Canberra: ABS, 2013. (ABS Cat. No. 3303.0.)

5 Stewart S, Ekman I, Ekman T, et al. Population impact of heart failure and the most common forms of cancer: a study of 1162309 hospital cases in Sweden (1988 to 2004). Circ Cardiovasc Qual Outcomes 2010; 3: 573-580.

6 Teng THK, Hung J, Knuiman M, et al. Trends in long-term cardiovascular mortality and morbidity in men and women with heart failure of ischemic versus non-ischemic aetiology in Western Australia between 1990 and 2005. Int J Cardiol 2012; 158: 405-410. doi: 10.1016/j.ijcard.2011.01.061.

7 Australian Institute of Health and Welfare. Separation statistics by principal diagnosis in ICD-10-AM, Australia, 1998-99 to 2007-08; 2011-12. http://www.aihw.gov.au/hospitals-data/principal-diagnosis-data-cubes (accessed Jul 2014)

8 Australian Institute of Health and Welfare. Cardiovascular disease: Australian facts 2011. Canberra: AlHW. 2011. (AlHW Cat. No. CVD 53; Cardiovascular Disease Series No. 35.)

9 Krum H, Abraham WT. Heart failure. Lancet 2009; 373: 941-955.

10 Davies J, Anderson J, Birdsey G, et al. Hospital Admission Risk Program (HARP): chronic heart failure working party report. Melbourne: Victorian Government Department of Human Services, 2003.

11 Robertson J, McElduff P. Pearson SA, et al. The health services burden of heart failure: an analysis using linked population health data-sets. BMC Health Serv Res 2012; 12: 103.

12 Westert GP, Lagoe RJ, Keskimaki I, et al. An international study of hospital readmissions and related utilization in Europe and the USA. Health Policy 2002; 61: 269-278.

13 Heller RF, Fisher JD, D'Este CA, et al. Death and readmission in the year after hospital admission with cardiovascular disease: the Hunter Area Heart and Stroke Register. Med J Aust 2000; 172: 261-265.

14 Driscoll A, Davidson P, Clark R, et al. Tailoring consumer resources to enhance self-care in chronic heart failure. Aust Crit Care 2009; 22: 133-140.

15 Driscoll A, Worrall-Carter L, McLennan S, et al. Heterogeneity of heart failure management programs in Australia. Eur J Cardiovasc Nurs 2006; 5: 75-82.

16 Driscoll A, Worrall-Carter L, Hare DL, et al. Evidence-based chronic heartfailure management programmes: reality or myth? BMJ Qual Saf 2011; 20: 31-37.

17 Krum H, Jelinek MV, Stewart S, et al; CHF Guidelines Core Writers. Guidelines for the prevention, detection and management of people with chronic heart failure in Australia 2006. Med J Aust 2006; 185: 549-556.

18 Woods JA, Katzenellenbogen JM, Davidson PM, Thompson SC. Heart failure among Indigenous Australians: a systematic review. BMC Cardiovasc Disord 2012; 12: 99.

19 Hauptman PJ, Rich MW, Heidenreich PA, et al. The heart failure clinic: a consensus statement of the Heart Faulure Society of America. J Card Fail 2008; 14: 801-815.

20 McAlister FA, Stewart S, Ferrua S, McMurray JJ. Multidisciplinary strategies for the management of heart failure patients at high risk for admission: a systematic review of randomized trials. J Am Coll Cardiol 2004; 44: 810-819.

21 Davidson P, Driscoll A, Huang N, et al. Multidisciplinary care for people with chronic heart failure: principles and recommendations for best practice. Melbourne: National Heart Foundation of Australia, 2010.

22 Driscoll A, Tonkin A, Stewart A, et al. Development of an evidence-based scoring system (HF-IS) to assess the quality of heart failure programmes for patients postdischarge from hospital. J Clin Nurs 2011; 20: 3011-3019.

23 Hanna A; Ontario Medical Association. OMA policy on patient-centred care. Ont Med Rev 2010; (Jun): 34-49. https://www.oma.org/Resources/ Documents/Patient-CentredCare,2010.pdf (accessed May 2013).

24 Hofmarcher MM, Oxley H, Rusticelli E. Improved health system performance through better care coordination. OECD health working paper No. 30. Paris: Organisation for Economic Co-operation and Development, 2007.

25 Bodenheimer T. Wagner EH, Grumbach K. Improving primary care for patients with chronic illness: the chronic care model, Part 2. JAMA 2002; 288: 1909-1914.

26 Glasgow RE, Funnell MM, Bonomi AE, et al. Self-management aspects of the improving chronic illness care breakthrough series: implementation with diabetes and heart failure teams. Ann Behav Med 2002: 24:80-87.
27 Komajda M, Hanon O, Hochadel M, et al. Contemporary management of octogenarians hospitalized for heart failure in Europe: Euro Heart Failure Survey II. Eur Heart J 2009; 30: 478-486.

28 Lainscak M, Cleland JG, Lenzen MJ, et al. International variations in the treatment and co-morbidity of left ventricular systolic dysfunction: data from the EuroHeart Failure Survey. Eur J Heart Fail 2007; 9: 292-299.

29 Groene O, Lombarts MJ, Klazinga N, et al. Is patient-centredness in European hospitals related to existing quality improvement strategies? Analysis of a cross-sectional survey (MARQulS study). Qual Saf Health Care 2009; 18 Suppl 1: i44-i50.

30 Australian Commission on Safety and Quality in Health Care. National Safety and Quality Health Service Standards, September 2012. Sydney: ACSQHC, 2012.

31 Krum H, Jelinek MV Stewart S, et al: National Heart Foundation of Australia and the Cardiac Society of Australia and New Zealand (Chronic Heart Failure Guidelines Expert Writing Panel). Guidelines for the prevention, detection and management of chronic heart failure in Australia. Updated October 2011. Melbourne: NHFA, 2011.

32 Takeda A, Taylor SJC, Taylor RS, et al. Clinical service organisation for heart failure. Cochrane Database Syst Rev 2012; (9): CD002752.

33 Riegel B, Moser DK, Anker SD, et al. State of the science: promoting self-care in persons with heart failure: a scientific statement from the American Heart Association. Circulation 2009; 120: 1141-1163.

34 Kansagara D, Englander $\mathrm{H}$, Salanitro A, et al. Risk prediction models for hospital readmission: a systematic review. JAMA 2011; 306: 1688-1698.

35 Clark RA, Driscoll A. Access and quality of heart failure management programs in Australia. Aust Crit Care 2009; 22: 111-116.

36 Institute for Patient- and Family-Centered Care. Advancing the practice of patient- and family-centered care in hospitals: how to get started. Bethesda, Md: IPFCC, 2011

37 Longtin Y, Sax H, Leape LL, et al. Patient participation: current knowledge and applicability to patient safety. Mayo Clin Proc 2010; 85: 53-62.

38 Stewart M, Brown JB, Donner A, et al. The impact of patient-centered care on outcomes. J Fam Pract 2000; 49: 796-804.

39 Agency for Healthcare Research and Quality (US). AHRQ Health Care Innovations Exchange. Patient- and family-centered care initiative is associated with high patient satisfaction and positive outcomes for total joint replacement patients. Rockville, Md: AHRQ, 2008 [updated 2013]. http://www.innovations.ahrq.gov/content.aspx?id=1764 (accessed May 2013).

40 Jha AK, Orav EJ, Zheng J, Epstein AM. Patients' perception of hospital care in the United States. N Engl J Med 2008; 359: 1921-1931.

41 Driscoll A, Tonkin A, Stewart A, et al. Complexity of management and health outcomes in a prospective cohort study of 573 heart failure patients in Australia: does more equal less? J Clin Nurs 2013; 22: 1629-1638.

42 New South Wales Department of Health. NSW clinical service framework for heart failure. Overview of the framework and its standards: volume 1. Sydney: NSW Department of Health, 2003.

43 Abrahamyan L, Boom N, Donovan LR, Tu JV; Canadian Cardiovascular Society Quality Indicators Steering Committee. An international environmental scan of quality indicators for cardiovascular care. Can J Cardiol 2012; 28: 110-118.

44 Stewart S, Horowitz JD. Home-based intervention in congestive heart failure: long-term implications on readmission and survival. Circulation 2002; 105: 2861-2866

45 Stewart S, Carrington MJ, Marwick T, et al. The WHICH? trial: rationale and design of a pragmatic randomized, multicentre comparison of home-vs. clinic-based management of chronic heart failure patients. Eur J Heart Fail 2011; 13: 909-916.

46 Productivity Commission. Australia's health workforce: research report. Canberra: Commonwealth of Australia, 2005.

47 Clark RA, Driscoll A, Nottage J, et al. Inequitable provision of optimal services for patients with chronic heart failure: a national geo-mapping study. Med J Aust 2007; 186: 169-173.

48 Segal L, Bolton T. Issues facing the future health care workforce: the importance of demand modelling. Aust New Zealand Health Policy 2009; 6: 12.

49 Australian Institute for Primary Care. System reform and development for chronic disease management. Melbourne: La Trobe University, 2008.

50 Driscoll A, Worrall-Carter L, Stewart S. Rationale and design of the National Benchmarking and Evidence-based National Clinical Guidelines for Chronic Heart Failure Management Programs Study. J Cardiovasc Nurs 2006; 21: 276-282. 